\title{
Facile Synthesis and Characterization of Novel CdS/BiOI Heterojunctions with Enhanced Visible-Light Photocatalytic Performances
}

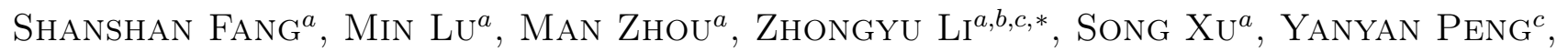 \\ QINCHI LI ${ }^{a, c}$ AND DAYONG $\mathrm{LU}^{c}$ \\ ${ }^{a}$ Changzhou University, Jiangsu Key Laboratory of Advanced Catalytic Materials and Technology, \\ School of Petrochemical Engineering, Changzhou 213164, PR China \\ ${ }^{b}$ Advanced Catalysis and Green Manufacturing Collaborative Innovation Center, \\ Changzhou University, Changzhou 213164, PR China \\ ${ }^{c}$ Jilin Institute of Chemical Technology, Jilin 132022, PR China \\ (Received September 1, 2016; in final form January 7, 2017)
}

\begin{abstract}
Novel CdS/BiOI heterojunction photocatalysts were successfully prepared by facile method. The as-prepared samples were characterized by transmission electron microscopy, field-emission scanning electron microscopy, Xray diffraction, the Raman spectroscopy, UV-vis diffuse reflectance spectra, the Fourier transform infrared spectra, and photoluminescence. It was found that CdS nanoparticles were uniformly distributed on the surface of BiOI microspheres. The photodegradation tests showed that the photocatalytic efficiency was increased at first and then decreased when further increasing CdS content in the nanocomposites. The highest activity was obtained by $3 \%$-CdS/BiOI nanocomposites. The enhanced photocatalytic performances were attributed to the matched band potentials of $\mathrm{CdS}$ and $\mathrm{BiOI}$, which resulted in the efficient separation of photogenerated electron-hole pairs. Based on the experimental results, a reasonable photocatalytic mechanism of CdS/BiOI photocatalysts was also proposed.
\end{abstract}

DOI: 10.12693/APhysPolA.131.263

PACS/topics: CdS/BiOI heterojunctions, visible-light photocatalyst, photocatalytic activity, nanocomposites

\section{Introduction}

Semiconductor photocatalysts have attracted considerable attention in recent years due to its wide application in pollutant degradation and hydrogen production from splitting water using solar energy $[1,2]$. Among various semiconductor materials, $\mathrm{TiO}_{2}$ semiconductor has been mostly investigated owing to the non-toxicity, low cost, relatively high chemical stability, and strong oxidizing power $[3,4]$. However, owing to its wide band gap $\left(E_{g}=3.2 \mathrm{eV}\right), \mathrm{TiO}_{2}$ requires ultraviolet light (UV) instead of more abundant visible light for effective photocatalysis [5]. Hence, developing visible-light-driven semiconductor photocatalysts is one of the most key topics for its application in environmental protection [6-10].

Recently, bismuth oxyhalides $\mathrm{BiOX}(\mathrm{X}=\mathrm{F}, \mathrm{Cl}, \mathrm{Br}$, and I) are a new category of promising photocatalysts, which manifests unusual visible-light photocatalytic activity for photocatalytic energy conversion and environment remediation, because of their unique anisotropic layered-structures, the internal static electric field, and strong absorption in the visible light region $[11,12]$. Among them, BiOI has the maximum absorption abi-

\footnotetext{
* corresponding author; e-mail: zhongyuli@mail.tsinghua.edu.cn
}

lity in visible-light region due to its smallest band gap $(\approx 1.8 \mathrm{eV})$ [13]. However, due to the low separation efficiency of photogenerated electrons and holes, the photocatalytic activity of $\mathrm{BiOI}$ is very poor [14-16].

A strategy for improving the photocatalytic efficiency of $\mathrm{BiOI}$ is to couple it with another semiconductor to form heterojunction at the interface [17]. Such as $\mathrm{TiO}_{2} / \mathrm{BiOI}[18,19], \mathrm{ZnO} / \mathrm{BiOI}[20], \mathrm{Bi}_{2} \mathrm{O}_{3} / \mathrm{BiOI}$ [21], $\mathrm{BiOBr} / \mathrm{BiOI}$ [22], $\mathrm{BiOCl} / \mathrm{BiOI}$ [23], $\mathrm{Pt} / \mathrm{BiOI}$ [24] and $\mathrm{AgI} / \mathrm{BiOI}[25]$ have been investigated as efficient visiblelight photocatalysts. The general concept is that the combined semiconductors should have different band gaps so that charge separation can be enhanced by the internal electric field driving force. CdS is a photocatalytic material with a high visible-light activity, due to its narrow band gap $(2.42 \mathrm{eV})[26]$. It has been widely used as a sensitizer to improve the visible-light response of wide band gap semiconductors [27]. Using CdS nanoparticles to decorate semiconductor materials can effectively separate the photogenerated electrons and holes, then result in an improvement of the photocatalytic efficiency [28].

Therefore, in the present study, the BiOI microspheres were synthesized by a facile solvothermal method. The $\mathrm{CdS} / \mathrm{BiOI}$ heterojunction photocatalysts were prepared by absorbing $\mathrm{CdS}$ nanoparticles on the surface of BiOI. The influence of CdS on the photocatalytic activity of $\mathrm{BiOI}$ was discussed, and the photocatalytic mechanism was proposed. 


\section{Experimental}

\subsection{Synthesis of the $C d S / B i O I$ heterojunctions}

All the reagents are analytical grade and used without further purification. BiOI powders were synthesized according to Ref. [29]. Briefly, $1.94 \mathrm{~g} \mathrm{Bi}\left(\mathrm{NO}_{3}\right)_{3} \cdot 5 \mathrm{H}_{2} \mathrm{O}$ and $0.830 \mathrm{~g} \mathrm{KI}$ were dissolved in $80 \mathrm{ml}$ ethylene glycol, respectively. Then KI solution was added into $\mathrm{Bi}\left(\mathrm{NO}_{3}\right)_{3}$ solution and the mixture was stirred for $10 \mathrm{~min}$ and then transferred into $200 \mathrm{ml}$ Teflon-lined stainless steel autoclave and maintained at $160^{\circ} \mathrm{C}$ for $6 \mathrm{~h}$. The products were washed for one time with ethanol and three times with distilled water and dried at $120^{\circ} \mathrm{C}$ for $8 \mathrm{~h}$.

To prepare $\mathrm{CdS}, 0.5187 \mathrm{~g} \mathrm{Cd}\left(\mathrm{CH}_{3} \mathrm{COO}\right)_{2} \cdot 2 \mathrm{H}_{2} \mathrm{O}$ and $0.1691 \mathrm{~g}$ thioacetamide (TAA) were dissolved in $15 \mathrm{ml}$ distilled water, respectively. Then TAA solution was added into $\mathrm{Cd}\left(\mathrm{CH}_{3} \mathrm{COO}\right)_{2} \cdot 2 \mathrm{H}_{2} \mathrm{O}$ solution and the mixture was stirred for $30 \mathrm{~min}$ to get a stable mixture. Then, a certain amount of polyvinylpyrrolidone (PVP) was put into the above mixture under stirring for another an hour and transferred into $50 \mathrm{ml}$ Teflon-lined autoclave. Finally, the autoclave was kept at $100^{\circ} \mathrm{C}$ for $12 \mathrm{~h}$ and natural cool down to room temperature. The resulting products were centrifuged, washed with ethanol and distilled water several times, and dried at $80^{\circ} \mathrm{C}$ for $8 \mathrm{~h}$.

The CdS/BiOI heterojunctions were prepared as following: an appropriate amount of solution containing the CdS was ultrasonicated for $30 \mathrm{~min}$ to make CdS totally dispersed. The as-prepared BiOI powder $(0.1 \mathrm{~g})$ was added into the above solution and stirred for $24 \mathrm{~h}$. The solvent was evaporated at $80^{\circ} \mathrm{C}$. The obtained powder was $\mathrm{CdS} / \mathrm{BiOI}$ heterojunctions. The mass ratio of $\mathrm{CdS}$ to $\mathrm{BiOI}$ was $1 \%, 3 \%$, and $5 \%$, respectively.

\subsection{Characterization}

To characterize the synthesized simples, X-ray diffraction (XRD), transmission electron microscope (TEM), field-emission scanning electron microscope (FESEM), the Fourier transform infrared spectra (FT-IR), photoluminescence (PL), the Raman and UV-vis absorption spectra were used. The crystalline catalyst was examined by XRD under a Rigaku D/Max-2500PC X-ray diffractometer (Rigaku Co., Japan) employing $\mathrm{Cu} K_{\alpha}$ radiation, $\lambda=1.54056$ Åoperated at $40 \mathrm{kV}$ and $100 \mathrm{~mA}$. A JEM-2100 TEM and a JSM-6360LA FE-SEM (JEOL, Japan) were used to characterize the morphology of the samples. FT-IR were recorded in the wave number range from 400 to $4000 \mathrm{~cm}^{-1}$ by Nicolet (PROTéGé 460) spectrometer. The Raman spectra were measured at room temperature using a LabRAM XploRA Raman spectrometer (Horiba Jobin Yvon, France) with a $532 \mathrm{~nm}$ laser focused on a spot about $3 \mu \mathrm{m}$ diameter. UV-vis diffuse reflectance spectroscopy (DRS) spectra of the photocatalysts were measured by a UV-vis scanning spectrophotometer (Shimadzu UV-2550) using an integrating sphere and $\mathrm{BaSO}_{4}$ as white standard. The PL spectra were obtained by an F-280 spectrometer (Tianjin Keqi, China).

\subsection{Adsorption experiments}

Batch experiments were performed by equilibrating $18 \mathrm{mg}$ of adsorbent with $30 \mathrm{ml}$ malachite green (MG) solutions of predetermined initial concentrations. $30 \mathrm{ml}$ of $V(V)$ solutions of concentration ranged from 4.0 to $16.0 \mathrm{mg} / \mathrm{l}$. By adding $\mathrm{NaOH}$ and $\mathrm{HCl}$ of different concentrations, initial $\mathrm{pH}$ of the solutions was maintained at required $\mathrm{pH}$ value. The suspensions were shaken for $1 \mathrm{~h}$. MG concentration was determined by UV759 UV-vis spectrophotometer at $617 \mathrm{~nm}$. The equilibrium sorption capacity was calculated using the following equation:

$$
q_{e}=C_{0}-C_{e} V / m,
$$

where $q_{e}$ is the equilibrium amount of $V(V)$ adsorbed per unit mass of adsorbent $[\mu \mathrm{mol} / \mathrm{g}], m$ is the sorbent mass [g], $V$ is the sample volume [L], $C_{e}$ is the equilibrium concentration $[\mathrm{mg} / \mathrm{L}]$ and $C_{0}$ is the initial concentration $[\mathrm{mg} / \mathrm{L}]$.

\subsection{Photocatalytic activity}

The photocatalytic activities of as-prepared products were evaluated by the degradation of MG solution. A $1 \mathrm{~kW}$ Xe lamp was used to simulate sunlight irradiation and a cutoff filter of $420 \mathrm{~nm}$ was used to filter the ultraviolet light. In a typical procedure, $0.03 \mathrm{~g}$ of as-prepared photocatalyst was dispersed into $50 \mathrm{ml}$ of $\mathrm{MG}$ solution $(15 \mathrm{mg} / \mathrm{l})$ and the suspension was magnetically stirred in the dark for $30 \mathrm{~min}$ to reach adsorption-desorption equilibrium between MG and photocatalyst. At every intervals of $20 \mathrm{~min}, 5 \mathrm{ml}$ of the suspension was collected, centrifuged to remove the photocatalyst particles. Finally, the catalyst-free MG solution was analyzed by a UV759 UV-vis spectrometer (Shanghai Precision \& Scientific Instrument Co., Ltd., China) to obtain the absorbance of the target contaminant at its maximum absorption wavelength. The decomposition efficiency of the target contaminant was calculated by $D=\left(1-C_{t} / C_{0}\right) \times 100 \%$, where $C_{0}$ and $C_{t}$ are the equilibrium concentration of the target contaminant before and after visible-light irradiation, respectively.

\section{Results and discussion}

\subsection{XRD patterns}

The detailed information on the crystal phase and crystallinity of the as-prepared samples was determined by XRD patterns. Figure 1 presents the XRD patterns of the pure $\mathrm{BiOI}$ and $\mathrm{CdS} / \mathrm{BiOI}$ nanocomposites with different CdS amounts. The pure BiOI displays sharp diffraction peaks (from $10 \circ$ to $75 \circ$ ) which are indexed to (012), (110), (013), (200), (114), (212), (115), (024), and (220) planes of the tetragonal phase structure (JCPDS card no. 73-2062). The XRD pattern of CdS with peaks around $26.7 \circ, 44.1 \circ$, and $52.2 \circ$ are shown in the inset of Fig. 1. In the diffraction pattern of $\mathrm{CdS} / \mathrm{BiOI}$ nanocomposites, they exhibit diffraction peaks very similar to those of the pure BiOI powders except for one small 


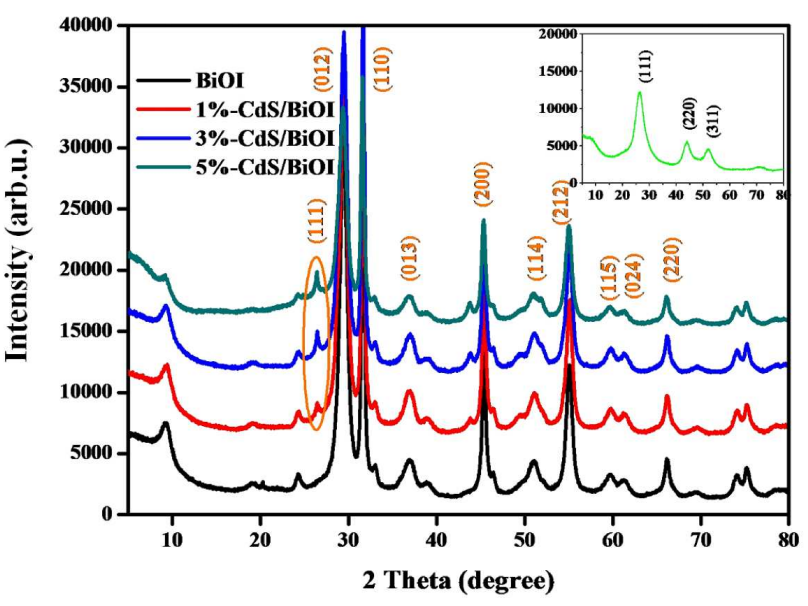

Fig. 1. XRD patterns of pure BiOI and series of CdS/BiOI nanocomposites.

peak at $26.7 \circ$ which is attributed to the characteristic peak of CdS. It can be clearly seen that the characteristic peaks of CdS become more intense with the increase of its loading amount. No impurity peaks were observed, confirming the high purity of the two products. The XRD results revealed the coexistence of BiOI and CdS in the $\mathrm{CdS} / \mathrm{BiOI}$ nanocomposites.

\subsection{FE-SEM images}

The FE-SEM morphologies and microstructures of $\mathrm{CdS}, \mathrm{BiOI}$, and $\mathrm{CdS} / \mathrm{BiOI}$ are presented in Fig. 2. The pure CdS shows very uniform particle morphology, and the particle size is less than $100 \mathrm{~nm}$ (Fig. 2a). The pure $\mathrm{BiOI}$ had 3-dimensional flower-like hierarchical architecture with the diameter of approximate 6-7 $\mu \mathrm{m}$ (Fig. 2b).

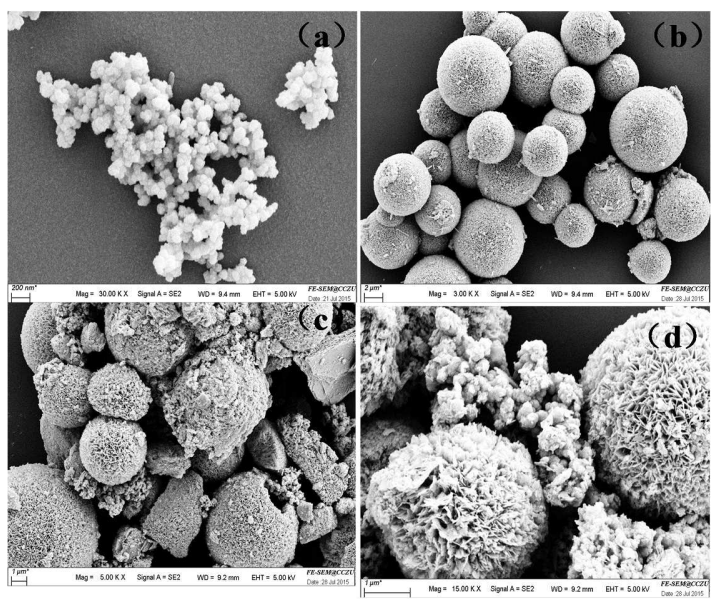

Fig. 2. FE-SEM images of the pure CdS (a), pure BiOI (b), and as-prepared 3\%-CdS/BiOI (c, d) nanocomposites.

It is obvious that $\mathrm{BiOI}$ hierarchical architecture was further composed of irregular nanoplates with thickness ranging from 50 to $100 \mathrm{~nm}$. It is obvious that BiOI hierarchi- cal architecture is further composed of irregular nanoplates with thickness ranging from 50 to $100 \mathrm{~nm}$. Figure 2c and $\mathrm{d}$ shows the SEM image of the $3 \%$-CdS/BiOI nanocomposite. The CdS nanoparticles were observed to be closely adhered to the surface of BiOI, resulting in intimate contact, which was thought to facilitate the transfer of photogenerated charges. The results indicated that the CdS nanoparticles can be easily attached to the surface of BiOI microspheres.

\subsection{TEM images}

Moreover, the crystal structure of the pure BiOI and the $3 \%$-CdS/BiOI heterojunction photocatalyst were further investigated by TEM and the HRTEM. Figure 3a shows the low-magnification TEM image of a single BiOI nanoflower. It can be clearly seen that the nanoflowers are self-assembled of nanosheets, which is consistent with the results of FE-SEM observation. From Fig. 3b, it can be seen that CdS nanoparticles closely adhered to

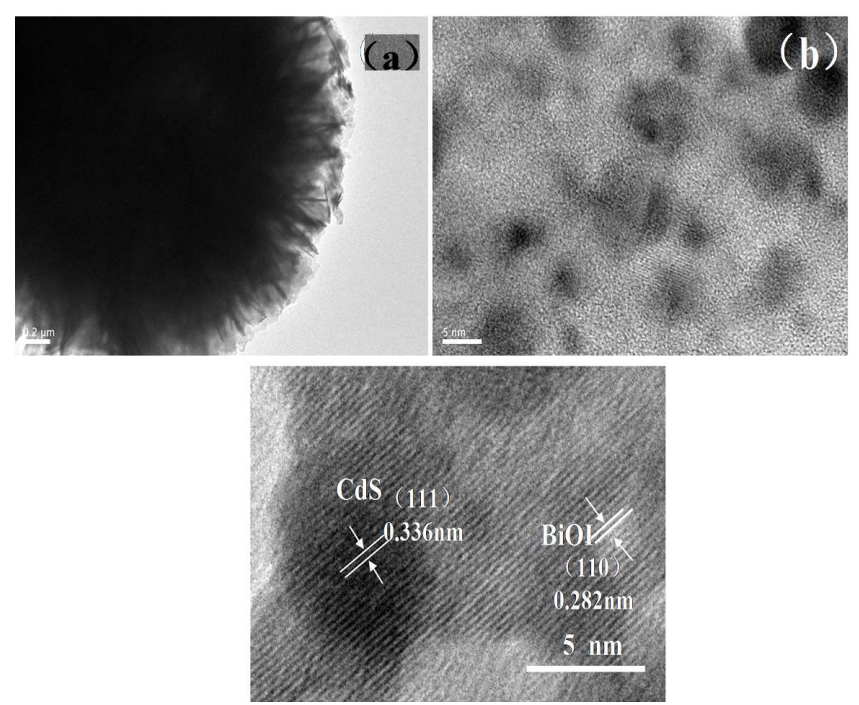

Fig. 3. TEM images of the pure BiOI (a), 3\%CdS/BiOI (b), and higher magnification TEM image of $3 \%$-CdS/BiOI nanocomposites (c).

the surface of the BiOI. The HRTEM image of the 3\%$\mathrm{CdS} / \mathrm{BiOI}$ photocatalyst was also displayed, as shown in Fig. 3c. The phase with the lattice fringes $(0.282 \mathrm{~nm})$ could be observed, which corresponds to the (110) plane of BiOI while the lattice space of crystallite locating on the $\mathrm{BiOI}$ is $0.336 \mathrm{~nm}$, which agrees well the (111) plane of CdS. The TEM result confirms assembling of CdS particles on the surface of the BiOI crystal.

\subsection{UV-vis diffuse reflectance spectra}

The optimum absorption property of photocatalyst and the migration of the light induced electrons and holes play a vital role in the photocatalytic process. Figure 4 shows the UV-vis DRS spectra of pure CdS, BiOI, and $\mathrm{CdS} / \mathrm{BiOI}$ heterostructure samples. It can be seen that 
all the samples show the intense absorption in the visiblelight region, which implies the possibility of photocatalytic activity under visible-light irradiation. For the pure CdS sample, the absorption edge occurs at a wavelength shorter than $570 \mathrm{~nm}$, whereas the absorption intensity of $\mathrm{BiOI}$ shows absorption edges at $630 \mathrm{~nm}$. In comparison to $\mathrm{CdS}$ and $\mathrm{BiOI}$, the absorption band edge of $\mathrm{CdS} / \mathrm{BiOI}$ exhibited an apparent red shift. This shift is ascribed to the interaction between $\mathrm{CdS}$ and BiOI semiconductors in the heterojunction. The enhanced light absorption of the nanocomposites led to the production of more photogenerated charge carriers and was thought to result in improved photocatalytic performance.

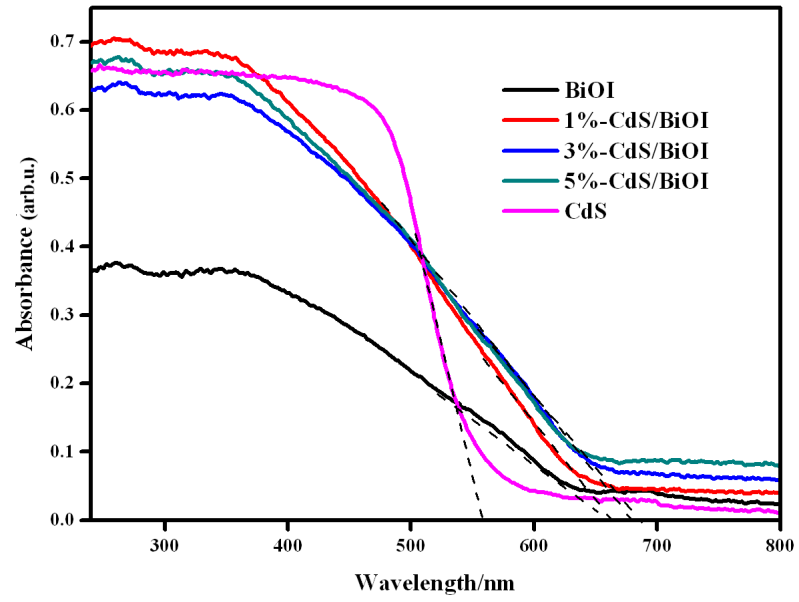

Fig. 4. UV-vis diffuse reflectance spectra of pure CdS, $\mathrm{BiOI}$, and series of $\mathrm{CdS} / \mathrm{BiOI}$ nanocomposites.

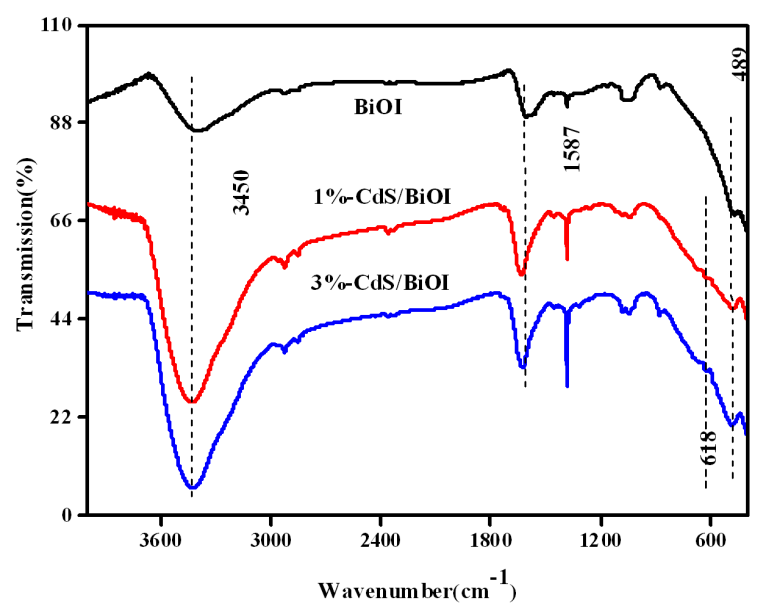

Fig. 5. FT-IR of pure BiOI and CdS/BiOI nanocomposites.

\subsection{FT-IR analysis}

To investigate the chemical composition and chemical bonding of composites, FT-IR investigation was carried out. Figure 5 displays the FT-IR spectra of pure
$\mathrm{BiOI}$ and $\mathrm{CdS} / \mathrm{BiOI}$ heterostructure samples. The broad bands centered at $3450 \mathrm{~cm}^{-1}$ and $1587 \mathrm{~cm}^{-1}$ in the spectrum of $\mathrm{BiOI}$ corresponded to the stretching and bending vibrations of $\mathrm{O}-\mathrm{H}$, respectively. Moreover, the band at $489 \mathrm{~cm}^{-1}$ for both samples of pure BiOI and $\mathrm{CdS} / \mathrm{BiOI}$ nanocomposites was associated with the stretching vibrations of the $\mathrm{Bi}-\mathrm{O}[30]$. In comparison with the spectrum of BiOI, the weak absorption band at $618 \mathrm{~cm}^{-1}$ could be ascribed to the $\mathrm{Cd}-\mathrm{S}$ group which was in the spectrum of the $\mathrm{CdS} / \mathrm{BiOI}$ nanocomposites. The peak ranged from 1120 to $1000 \mathrm{~cm}^{-1}$ could be attributed to the $\mathrm{C}-\mathrm{O}$ of ethanol which might come from the washing process. Absorption bands of $\mathrm{CdS}$ and $\mathrm{BiOI}$ were apparent in the $\mathrm{CdS} / \mathrm{BiOI}$ nanocomposites, confirming that $\mathrm{CdS}$ and BiOI had been coupled together successfully.

\subsection{Raman analysis}

The Raman scattering is an effective technique to investigate the crystallization, structure, and defect of nanostructure materials. Figure 6 shows the Raman spectra of $\mathrm{CdS}, \mathrm{BiOI}$, and $\mathrm{CdS} / \mathrm{BiOI}$ nanocomposites. The Raman spectra of CdS and CdS/BiOI nanocomposites displayed one prominent peak around at $148 \mathrm{~cm}^{-1}$.

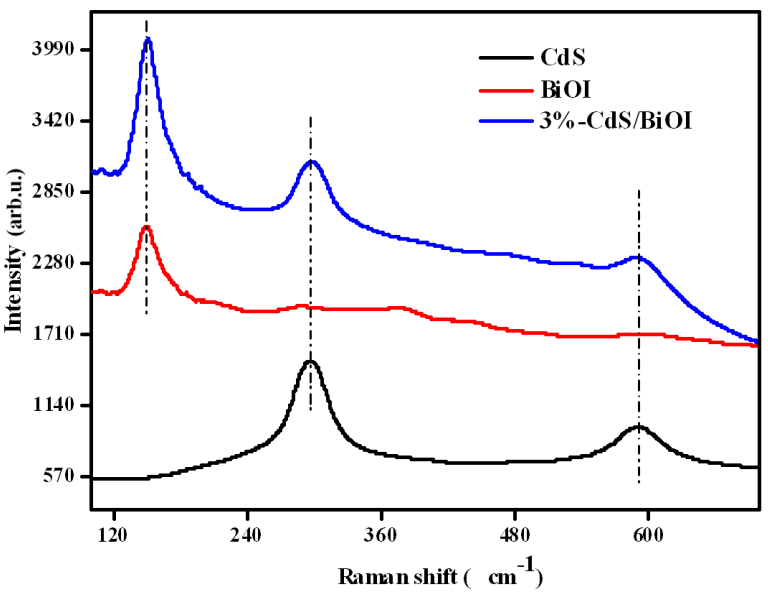

Fig. 6. Raman spectra of as-prepared BiOI, CdS, and $3 \%$-CdS/BiOI nanocomposites.

BiOI can be recognized based on the characteristic peak position of $148 \mathrm{~cm}^{-1}$ due to the $E_{g}$ internal Bi-I stretching mode. The curves of $\mathrm{CdS}$ and $\mathrm{CdS} / \mathrm{BiOI}$ exhibited two Raman active peaks. One well defined intense peak appeared at $295 \mathrm{~cm}^{-1}$ and a low intense peak at around $589 \mathrm{~cm}^{-1}$ was attributed to first and second order longitudinal-optical (LO) phonon modes.

\subsection{Adsorption experiments}

The adsorption isotherms of MG dye onto heterojunction surface was studied by taking solutions in the concentration ranging from 4.0 to $16.0 \mathrm{mg} / \mathrm{l}$ and the results are shown in Fig. 7. With increase of initial concentration of MG from 4.0 to $16.0 \mathrm{mg} / \mathrm{l}$, the adsorption capacity increased, indicating that $\mathrm{MG}$ adsorption depends on initial concentration. The result suggests that 


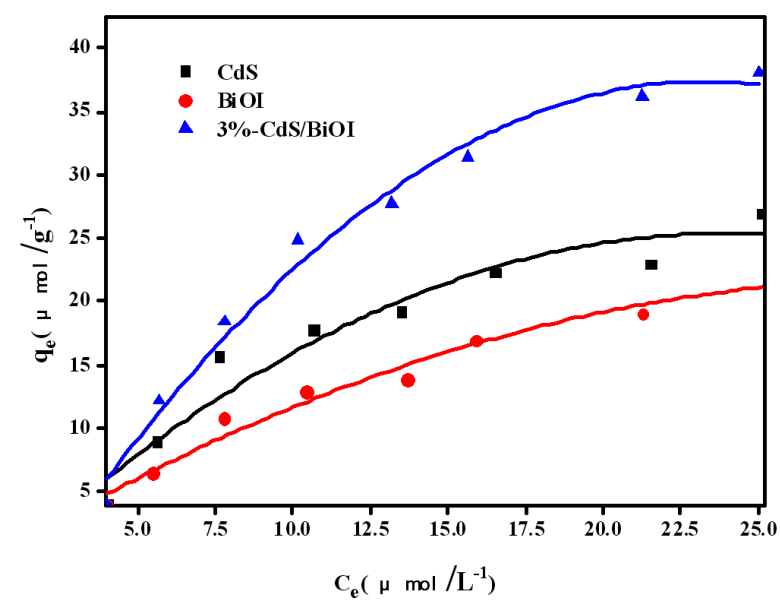

Fig. 7. Adsorption isotherms of $\mathrm{MG}$ on the CdS, BiOI, and $3 \%$-CdS/BiOI surfaces.

the doing of CdS could improve its adsorption abilities. The enhanced dye removal efficiency was attributed to the synergetic effects of the adsorption and photodegradation [31, 32].

\subsection{Photocatalytic activity of $\mathrm{CdS} / \mathrm{BiOI}$ nanocomposites}

The photocatalytic activities of $\mathrm{CdS} / \mathrm{BiOI}$ samples were measured by the degradation of MG under visible light irradiation. Figure 8a shows the change of absorption peak spectra of MG solution exposed to visible light as a function of time in the presence of $3 \%$-CdS/BiOI sample. During the photodegradation process, the intensity of the typical adsorption peak of MG at $617 \mathrm{~nm}$ decreased gradually as the photocatalytic reaction time increased, indicating the excellent photocatalytic activity of $3 \%$-CdS/BiOI nanocomposites for the MG degradation under visible-light irradiation. The larger part (92\%) of MG could be discolored by 3\%-CdS/BiOI nanocomposites within 100 min of visible-light irradiation. No new absorption bands appeared, indicating the complete photocatalytic degradation of MG during that reaction.

Figure $8 \mathrm{~b}$ shows the MG photodegradation over pure $\mathrm{CdS}, \mathrm{BiOI}$, and $\mathrm{CdS} / \mathrm{BiOI}$ heterostructures. It can be seen that the single $\mathrm{CdS}$ and pure BiOI exhibit weak degrade efficiency of $31.5 \%$ and $25.3 \%$ after $100 \mathrm{~min}$ of visible-light illumination and all of the $\mathrm{CdS} / \mathrm{BiOI}$ heterostructures exhibited superior photocatalytic activity. With increasing CdS content, the photocatalytic activity enhanced gradually and then decreased. As the content of CdS was $3 \%$, the highest photocatalytic activity could be achieved. These results suggest that $3 \% \mathrm{CdS}$ is the optimal sample among the CdS/BiOI nanocomposites, which can be mainly ascribed to the heterojunction structure between CdS and BiOI. With increasing CdS content, more $\mathrm{CdS} / \mathrm{BiOI}$ heterojunctions interface will be formed, which suppresses the recombination of photoinduced electron-hole pairs. However, the excessive CdS
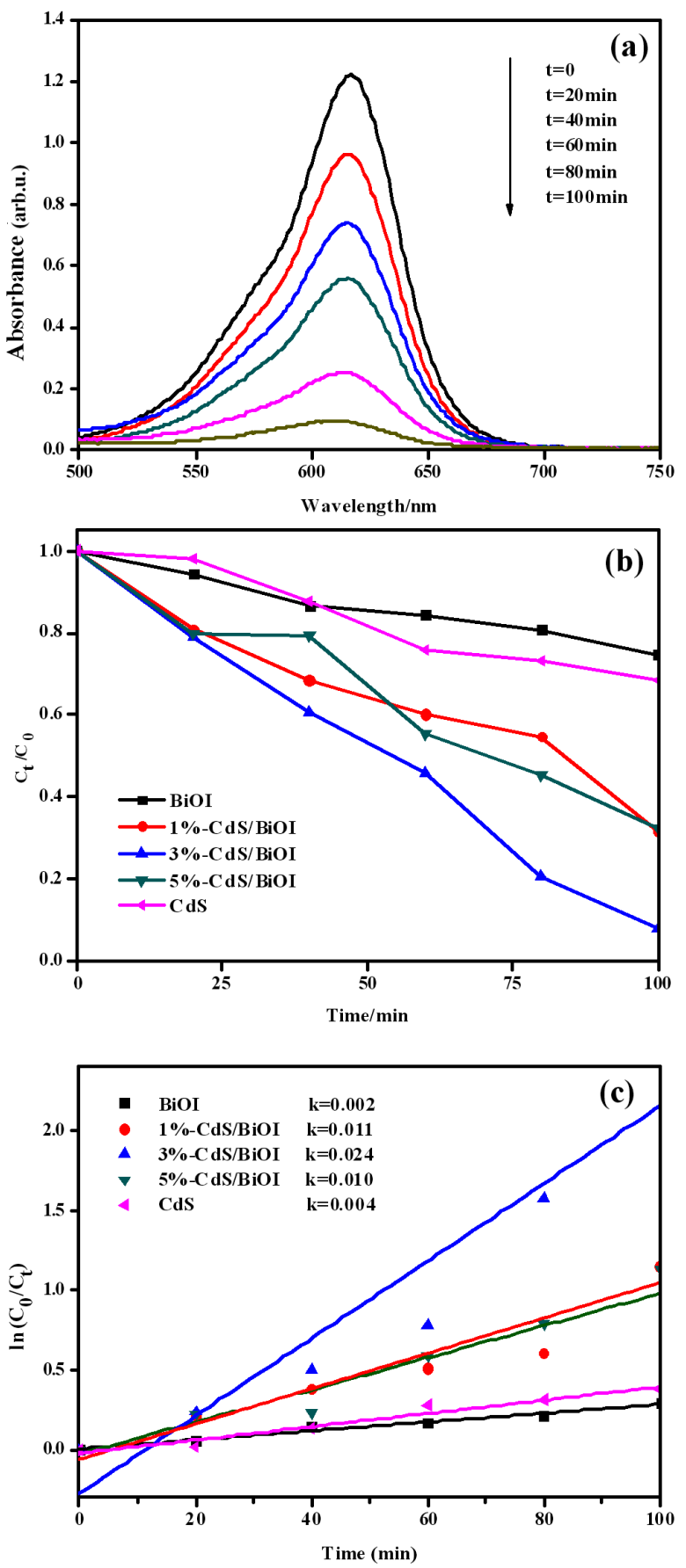

Fig. 8. (a) Temporal evolution of the spectra during the photodegradation of MG over $3 \%$-CdS/BiOI sample, (b) photodegradation of MG with pure CdS, BiOI and $\mathrm{CdS} / \mathrm{BiOI}$ nanocomposites under visible light irradiation, (c) kinetic data for the degradation of $\mathrm{MG}$ in the presence of different photocatalysts.

content will lead to the decrease of heterojunction interface and function as recombination centers of electronhole pairs. Consequently, suitable content of CdS could improve the photocatalytic activity.

Figure $8 \mathrm{c}$ reveals that the photocatalytic degradation of MG on different catalysts fits pseudo-first-order kine- 
tics, $\ln \left(C_{0} / C_{t}\right)=k_{a p p} t$, where $t$ is the irradiation time and $k_{a p p}$ is the rate constant. Moreover, the $k_{a p p}$ for MG degradation with $\mathrm{CdS}, \mathrm{BiOI}, 1 \%-\mathrm{CdS} / \mathrm{BiOI}, 3 \%-$ $\mathrm{CdS} / \mathrm{BiOI}$ and $5 \%-\mathrm{CdS} / \mathrm{BiOI}$ were estimated to be 0.004 , $0.002,0.011,0.024$, and $0.010 \mathrm{~min}^{-1}$, respectively. It is worth mentioning that the $\mathrm{MG}$ degradation rate constant of the $\mathrm{CdS} / \mathrm{BiOI}$ nanocomposites is much higher than that of $\mathrm{CdS}$ and BiOI. Remarkably, the rate constant of the $3 \%$-CdS/BiOI nanocomposite is 12 times as high as that of pure BiOI under the same experimental conditions, which indicates that the coupling of CdS could enhance the photocatalytic capability of BiOI under the visible-light irradiation.

\subsection{Photoluminescence analysis}

PL spectra are usually used to estimate the separation efficiency of photoinduced charge carriers for semiconductors. A higher intensity of PL indicates a faster recombination of charge carriers. Figure 9 shows the PL spectra of the as-prepared samples at an excitation wavelength of 300 nm. Pure CdS, BiOI, and CdS/BiOI nanocomposites exhibit the similar peak shape and peak position, but the intensities of peaks are different with each other. In contrast, the emission intensity from $\mathrm{CdS} / \mathrm{BiOI}$ nanocomposites is clearly reduced, which suggests that the recombination of photogenerated electrons and holes is effectively inhibited. The intensity of the $3 \%$-CdS/BiOI nanocomposites photocatalyst was the lowest than other photocatalysts, which indicated that the $3 \%-\mathrm{CdS} / \mathrm{BiOI}$ nanocomposites had a lowest recombination rate of photogenerated charge carriers. Therefore, the heterostructured $\mathrm{CdS} / \mathrm{BiOI}$ nanocomposites are highly favorable for the efficient separation of the photo-induced charge carriers during the photocatalytic reactions.

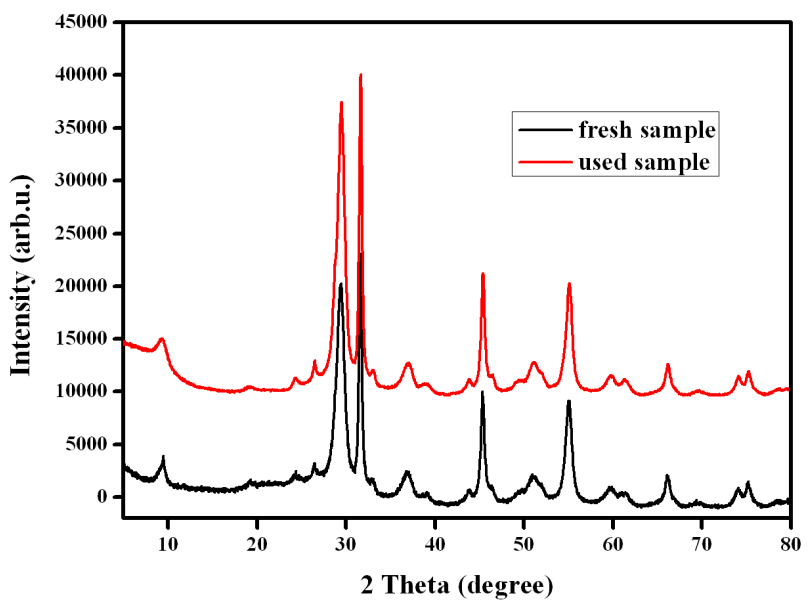

Fig. 9. PL spectra of pure CdS, pure BiOI and series of $\mathrm{CdS} / \mathrm{BiOI}$ nanocomposites.

\subsection{Photocatalytic mechanism}

The reusability and durability are important parameters to explore real applications of catalysts. As a result, $3 \%$-CdS/BiOI nanocomposites were specifically chosen for successive catalytic estimations in Fig. 10. There is no obvious decrease of photocatalytic efficiency over MG for five cycling runs under visible light, possibly linking to the well-maintained chemical composition and phase structures.

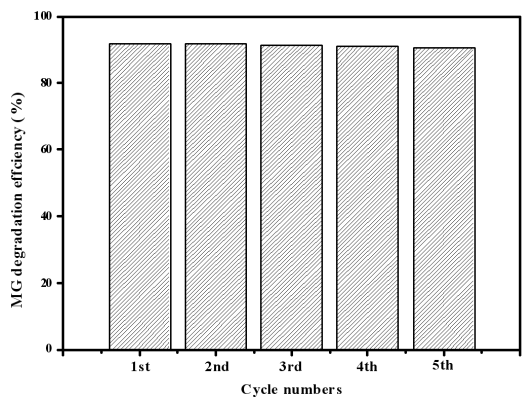

Fig. 10. The stability of $3 \%$-CdS/BiOI photocatalyst over MG degradation.

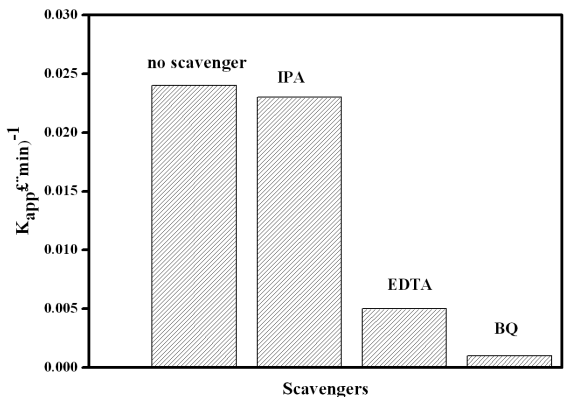

Fig. 11. Effects of different scavengers on the degradation of $\mathrm{MG}$ in the presence of $3 \%-\mathrm{CdS} / \mathrm{BiOI}$ under visible-light irradiation.

In the photodegradation process of organic pollutants, reactive species as superoxide radical $\left(\cdot \mathrm{O}_{2}^{-}\right)$, hole $\left(h^{+}\right)$ and hydroxyl radical $(\cdot \mathrm{OH})$ acted as a bridge, playing the role of photocatalysts under the light irradiation, therefore, it is necessary to determine the main reactive species for the degradation of MG in this study. Some sacrificial agents, such as iso-propyl alcohol (IPA), disodium ethylenediaminetetraacetate (EDTA) and 1,4-benzoquinone (BQ) were used as the scavengers of $\cdot \mathrm{OH}, h^{+}$and $\cdot \mathrm{O}_{2}^{-}$, respectively. Figure 11 shows that the photodegradation efficiency of MG over $3 \%$-CdS/BiOI is not affected by the addition of IPA, indicating that almost no superoxide radicals $(\cdot \mathrm{OH})$ were involved in the degradation of MG. When EDTA was added, the photodegradation efficiency of MG obviously decreased, which revealed the existence of $h^{+}$radical species. Besides, the photocatalytic activity was thoroughly suppressed by addition of $\mathrm{BQ}$, suggesting that the $\cdot \mathrm{O}_{2}^{-}$pathways play a crucial role in the process of MG degradation. These results indicated that the $h^{+}$and $\cdot \mathrm{O}_{2}^{-}$were the main active species in the MG photodegradation process.

The $E_{g}$ values of pure $\mathrm{BiOI}$ and $\mathrm{CdS}$ were estimated to be $1.8 \mathrm{eV}$ and $2.42 \mathrm{eV}$ in the optical property section. 
The valence band (VB) edge potential of a semiconductor can be predicted by the following empirical equation [3335]:

$$
E_{C B}=X-E^{e}-0.5 E_{g},
$$

where $X$ is the absolute electronegativity of the semiconductor, $E^{e}$ is the energy of free electrons on the hydrogen scale $(4.5 \mathrm{eV}), E_{g}$ is the band gap of the semiconductor. The $E_{V B}$ value could be deduced from the equation [36]:

$$
E_{V B}=E_{C B}+E_{g} \text {. }
$$

It could be seen that the calculated positions of $\mathrm{CB}$ and VB edge potentials of $\mathrm{BiOI}$ were determined to be $0.6 \mathrm{eV}$ and $2.4 \mathrm{eV}$, respectively. For CdS, the $\mathrm{CB}$ and VB potentials of CdS were determined to be $-0.5 \mathrm{eV}$ and $1.92 \mathrm{eV}$, respectively.

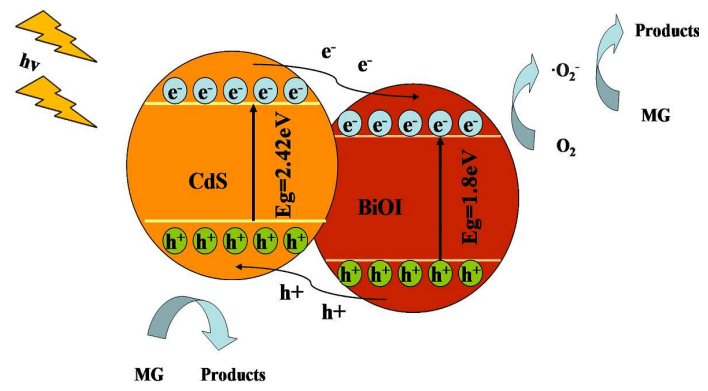

Fig. 12. Proposed mechanism for the photodegradation of MG over CdS/BiOI nanocomposites under visible light irradiation.

Based on the above analysis, the possible mechanism for the photocatalytic activity enhancement of $3 \%$ $\mathrm{CdS} / \mathrm{BiOI}$ nanocomposite is proposed in Fig. 12. Under visible-light irradiation, $\mathrm{CdS}$ and $\mathrm{BiOI}$ would be excited simultaneously and generate electron-hole pairs. On the one hand, the $\mathrm{CB}$ electrons (CdS) easily flow into the $\mathrm{CB}$ edge of $\mathrm{BiOI}$ through the interface because the $\mathrm{CB}$ edge of CdS is higher than that of BiOI. On the other hand, because the VB edge level of $\mathrm{BiOI}$ is lower than that of $\mathrm{CdS}$, holes in the VB edge of BiOI will transfer to that of CdS by the control of the interface. In such way, photoinduced electron-hole pairs could be separated efficiently at the interface of two semiconductors. Consequently, the photogenerated electrons could easily transfer from the reformed $\mathrm{CB}$ of $\mathrm{CdS}$ to that of $\mathrm{BiOI}$, and then react with $\mathrm{O}_{2}$ to generate reactive $\cdot \mathrm{O}_{2}^{-}$radicals. Meanwhile the holes can also move in the opposite direction from the $\mathrm{VB}$ of BiOI to that of CdS. Therefore the $h^{+}$and $\cdot \mathrm{O}_{2}^{-}$radicals were beneficial for the MG degradation. As a result, efficient separation of photogenerated electronhole pairs is achieved by the distribution of electrons and holes on different semiconductors and thus enhanced the corresponding photocatalytic performance.

In addition, the XRD patterns of the sample before and after the reaction (Fig. 13) showed that the crystal structure had little change and no additional band appeared during the photodegradation process. These suggest that

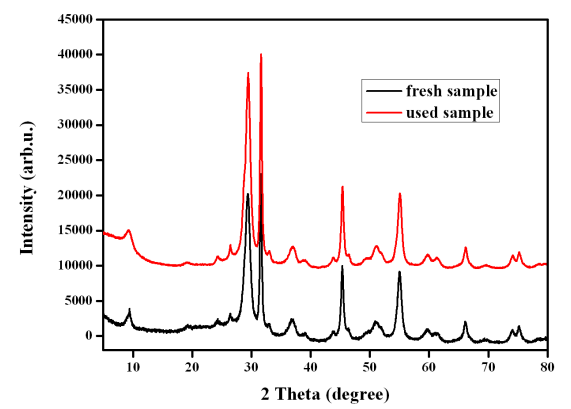

Fig. 13. XRD pattern of the $3 \%-\mathrm{CdS} / \mathrm{BiOI}$ before and after the long time experiment.

the nanocomposite was not photocorroded and presented relatively high stability.

\section{Conclusion}

CdS/BiOI heterojunctions have been successfully prepared by a facile method. The CdS nanoparticles were uniformly distributed on the surface of BiOI microspheres. The nanocomposites exhibited good visible-light absorption. The PL results revealed that the recombination of photogenerated electrons and holes was inhibited. The highest activity was obtained by $3 \%$-CdS $/ \mathrm{BiOI}$ heterojunctions. The enhanced photocatalytic performance was ascribed to the improved photogenerated carrier separation efficiency caused by the coupling effects of the two semiconductors. The as-prepared CdS/BiOI heterostructure photocatalysts might have potential in environmental remediation applications.

\section{Acknowledgments}

This work was financially supported by Open Fund of Key Laboratory of Regional Environment and Ecoremediation of Ministry of Education, China (SYU-KFE-12), Open Fund of Key Laboratory of Contaminated Environment Control and Regional Ecology Safety, Shenyang University (SYU-KF-L-12), Natural Science Foundation of Jiangsu Province, China (No. BK20150259) and Natural Science Foundation of Changzhou City (CJ20140053).

\section{References}

[1] C.C. Chen, W.H. Ma, J.C. Zhao, Chem. Soc. Rev. 39, 4206 (2010).

[2] F. Zhao, Q.F. Lu, Z.L. Xiu, C.F. Zhu, Nano 11, 1650048 (2016).

[3] H. Liu, T. Lv, C.K. Zhu, X. Su, Z.F. Zhu, J. Mol. Catal. A Chem. 396, 136 (2015).

[4] B. Zeng, X.H. Chen, X.T. Ning, C.S. Chen, H. Long, Nano 8, 1350052 (2013).

[5] Q.J. Xiang, J.G. Yu, W.G. Wang, M. Jaroniec, Chem. Commun. 47, 6906 (2011).

[6] Y.J. Wang, R. Shi, J. Lin, Y.F. Zhu, Energy Environ. Sci. 4, 2922 (2011). 
[7] H.C. Zhang, H. Huang, H. Ming, H.T. Li, L.L. Zhang, Y. Liu, Z.H. Kang, J. Mater. Chem. 22, 10501 (2012).

[8] R. Azimirad, S. Safa, O. Akhavan, Acta Phys. Pol. A 127, 1727 (2015).

[9] A. Naldoni, M. Allieta, S. Santangelo, M. Marelli, F. Fabbri, S. Cappelli, C.L. Bianchi, R. Psaro, V.D. Santo, J. Am. Chem. Soc. 134, 7600 (2012).

[10] M.A. Ischay, M.E. Anzovino, J. Du, T.P. Yoon, J. Am. Chem. Soc. 130, 12886 (2008).

[11] J. Li, Y. Yu, L.Z. Zhang, Nanoscale 6, 8473 (2014).

[12] T. Yan, H.Y. Liu, P.C. Gao, M. Sun, Q. Wei, W.G. Xu, X.D. Wang, B. Du, New J. Chem. 39, 3964 (2015)

[13] X.F. Chang, J. Huang, Q.Y. Tan, M. Wang, G.B. Ji, S.B. Deng, G. Yu, Catal. Commun. 10, 1957 (2009).

[14] J. Cao, B.Y. Xu, H.L. Lin, S.F. Chen, Chem. Eng. J. 228, 482 (2013).

[15] J. Cao, B.Y. Xu, H.L. Lin, B.D. Luo, S.F. Chen, Dalton Trans. 41, 11482 (2012).

[16] H.Q. Li, Y.M. Cui, W.S. Hong, Appl. Surf. Sci. 264 , $581(2013)$.

[17] Z.K. Cui, M.M. Si, Z. Zheng, L.W. Mi, W.J. Fa, H.M. Jia, Catal. Commun. 42, 121 (2013).

[18] X. Zhang, L.Z. Zhang, T.F. Xie, D.J. Wang, J. Phys. Chem. C 113, 7371 (2009).

[19] G.P. Dai, J.G. Yu, G. Liu, J. Phys. Chem. C 115, 7339 (2011)

[20] J. Jiang, X. Zhang, P.B. Sun, L.Z. Zhang, J. Phys. Chem. C 115, 20555 (2011).

[21] Y.Y. Li, J.S. Wang, H.C. Yao, L.Y. Dang, Z.J. Li, Catal. Commun. 12, 660 (2011).
[22] J. Cao, B.Y. Xu, B.D. Luo, H.L. Lin, S.F. Chen, Catal. Commun. 13, 63 (2011).

[23] T.B. Li, G. Chen, C. Zhou, Z.Y. Shen, R.C. Jin, J.X. Sun, Dalton Trans. 40, 6751 (2011).

[24] C.L. Yu, J.C. Yu, C.F. Fan, H.R. Wen, S.J. Hu, Mater. Sci. Eng. B 166, 213 (2010).

[25] H.F. Cheng, B.B. Huang, Y. Dai, X.Y. Qin, X.Y. Zhang, Langmuir 26, 6618 (2010).

[26] D.W. Jing, L.J. Guo, J. Phys. Chem. C 111, 13437 (2007).

[27] D.W. Jing, L.J. Guo, J. Phys. Chem. B 110, 11139 (2006).

[28] N.Z. Bao, L.M. Shen, T. Takata, K. Domen, Chem. Mater. 20, 110 (2008).

[29] Y.F. Liu, W.Q. Yao, D. Liu, R.L. Zong, M. Zhang, X.G. Ma, Y.F. Zhu, Appl. Catal. B 163, 547 (2015).

[30] H.L. Lin, C.C. Zhou, J. Cao, S.F. Chen, Chin. Sci. Bull. 59, 3420 (2014).

[31] A. Turki, C. Guillard, F. Dappozze, Z. Ksibi, G. Berhault, H. Kochkar, Appl. Catal. B 163, 404 (2015).

[32] T.S. Anirudhan, F.J. Christa, J. Hazard. Mater. 324, 117 (2017).

[33] C. Chang, L. Zhu, S. Wang, X. Chu, L. Yue, ACS Appl. Mater. Interf. 6, 5083 (2014).

[34] Y. Sun, W. Zhang, T. Xiong, Z. Zhao, F. Dong, R. Wang, W. Ho, 0.1016/j.jcis.2013.12.037 J. Coll. Interf. Sci. 418, 317 (2014).

[35] F. Chang, C.L. Li, J. Chen, J. Wang, J.R. Luo, Y.C. Xie, B.Q. Deng, X.F. Hu, Superlatt. Microstruct. 76, 90 (2014).

[36] Z.C. Yang, J. Li, F.X. Cheng, Z. Chen, X.P. Dong, J. Alloys Comp. 634, 215 (2015). 